\title{
PH'11 best paper award by JAISE
}

During the 5th edition of the International Conference on Pervasive Computing Technologies for Healthcare (Pervasive Health 2011), held during 23-26 May 2011 in Dublin, Ireland http://pervasivehealth.org (event was sponsored by ICST and co-sponsored by CREATE-NET), JAISE provided a prize to the best paper of the conference. The prize for the best paper consists of one year free subscription to JAISE and other products accessible through MetaPress kindly provided by IOS Press, the publisher of JAISE.

The chairs of the conference directly based upon the scores and feedback provided by the Program Committee members during the review process awarded the prize to the paper:

"Evaluation of an Inexpensive Depth Camera for Passive In-Home Fall Risk Assessment" by Erik Stone and Marjorie Skubic (USA).
The photo in Fig. 1 shows Juan Carlos Augusto (coeditor in chief of JAISE) congratulating the first author on behalf of JAISE.

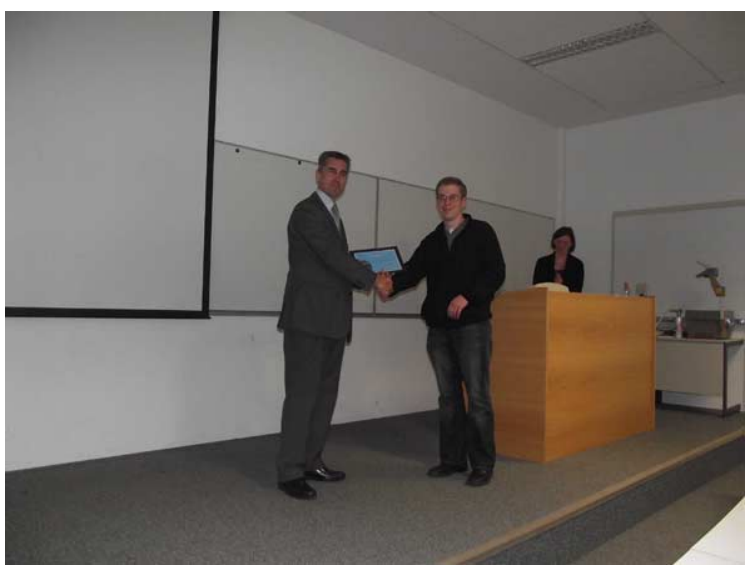

Fig. 1. JAISE congratulates the winner. 